\title{
HUBBLE SPACE TELESCOPE IMAGING OF THE WR 38/WR 38a CLUSTER ${ }^{1}$
}

\author{
Debra J. Wallace ${ }^{2}$ and Douglas R. Gies \\ Center for High Angular Resolution Astronomy, Department of Physics and Astronomy, Georgia State University, P.O. Box 4106, \\ Atlanta, GA 30302-4106; wallace@ $@$ chara.gsu.edu, gies@chara.gsu.edu \\ Anthony F. J. Mofate \\ Département de Physique and Observatoire du Mont Mégantic, Université de Montréal, CP 6128, Succursale Centre-Ville, \\ Montreal, QC H3C 3J7, Canada; moffat@astro.umontreal.ca \\ Michael M. Shara \\ Department of Astrophysics, American Museum of Natural History, New York, NY 10024; mshara@amnh.org \\ AND \\ VIRPI S. NIEMELA ${ }^{3}$ \\ Facultad de Ciencias Astronómicas y Geofísicas, Universidad Nacional de La Plata, Paseo del Bosque s/n, 1900 La Plata, Argentina; \\ virpi@lilen.fcaglp.unlp.edu.ar \\ Received 2004 April 12; accepted 2005 March 30
}

\begin{abstract}
We are conducting a high angular resolution imaging survey of Galactic Wolf-Rayet stars using the Wide Field Planetary Camera 2 aboard the Hubble Space Telescope (HST). We have found a small stellar cluster associated with the faint, close pair WR 38 and WR 38a. We present astrometric measurements and photometry in the wideband F336W $(U)$, F439W $(B)$, and F555W $(V)$ filter system for the cluster and nearby stars. We combine our photometry with Johnson and IR magnitudes and compare the observations with calibrated model results for reddened stars to adjust the HST zero points and to identify five probable main-sequence members of the cluster. A least-squares fit of the colors and magnitudes of this set yields a cluster reddening of $E(B-V)=1.63 \pm 0.05 \mathrm{mag}$ and a distance of $10_{-4}^{+12} \mathrm{kpc}$ for an assumed ratio of total to selective extinction of $R=3.1$. We discuss the relationship of this cluster to other objects along the line of sight. If situated at a distance of $\approx 8 \mathrm{kpc}$, then the cluster would reside in a dense region of the Carina spiral arm, close to a giant molecular cloud and the starburst cluster NGC 3603.
\end{abstract}

Key words: binaries: visual — open clusters and associations: individual (C1104-610a) — stars: imaging — stars: individual (WR 38, WR 38a) - stars: Wolf-Rayet

\section{INTRODUCTION}

Wolf-Rayet (WR) stars are the evolved, He-burning cores and hot stellar envelopes of once-massive O-type stars. Their strong winds (terminal velocities $v_{\infty} \approx 1000-2500 \mathrm{~km} \mathrm{~s}^{-1}$ ) and heavy mass-loss rates $\left[\dot{M} \simeq(1-6) \times 10^{-5} M_{\odot} \mathrm{yr}^{-1}\right.$; Willis 1991; Nugis \& Lamers 2002] power their characteristic strong, broad emission lines of $\mathrm{He}, \mathrm{N}, \mathrm{C}$, and $\mathrm{O}$, which correspond to increasingly evolved evolutionary states. While the environment and multiplicity of O-type stars have been explored in depth (Gies 1987; Mason et al. 1998), these characteristics for the evolved WR phase are not as well determined. In 1996 we initiated a survey with the Hubble Space Telescope Wide Field Planetary Camera 2 (HST WFPC2) to discover and quantify the multiplicity and environments of Galactic WR stars (Wallace 2003). Here we discuss two of our targets, WR 38 and WR 38a, that were revealed to be both multiple and contained within a small stellar cluster (Wallace et al. 1999).

WR 38 is a WC4-type star (MacConnell \& Sanduleak 1970), and WR 38a is a WN5-type star (Shara et al. 1991). The two are

\footnotetext{
${ }^{1}$ Based on observations with the NASA/ESA Hubble Space Telescope, obtained at the Space Telescope Science Institute, which is operated by the Association of Universities for Research in Astronomy (AURA), Inc., under NASA contract NAS5-26555.

${ }^{2}$ Current address: NASA Goddard Space Flight Center, Code 685, Building 21, Greenbelt, MD 20771; wallace@exol.gsfc.nasa.gov.

${ }^{3}$ Member of Carrera del Investigador, CIC-BA, Argentina.
}

separated by only $20^{\prime \prime}$ in the sky, and they are neighbors to another even tighter $5^{\prime \prime}$ pair of WR stars, WR $38 \mathrm{~b}$ and WR 39 , at a separation of 3.' 8 . These four plus WR 37 are surrounded by a ring nebula (Marston 1997), although it is not clearly associated with any one of these stars. Our view in this direction in the Galactic plane cuts through the Sagittarius-Carina spiral arm at distances of approximately 2.4 and $8 \mathrm{kpc}$ (Grabelsky et al. 1988; Georgelin et al. 2000; Russeil 2003). The photometric distances of these WR stars are generally close to $5 \mathrm{kpc}$ (van der Hucht 2001), which would place them among the young population of the Carina spiral arm. However, in a recent paper Shorlin et al. (2004) presented photometry for the stars in the immediate vicinity of WR 38 and WR 38a that indicated a much greater distance of $14.5 \pm 1.6 \mathrm{kpc}$. This distance would place the pair in an extension of the outer Perseus arm some $14 \mathrm{kpc}$ from the Galactic center. There are only four of 227 known Galactic WR stars that have such a great distance from the Galactic center (van der Hucht 2001). Shorlin et al. (2004) designate the cluster as C1104-610a, and images of the field surrounding the cluster are presented in Wramdemark (1976, Plate 4), van der Hucht et al. (1981), and Shorlin et al. (2004, Figs. 1 and 2).

Here we present new astrometric and photometric results on the stars found close to WR 38 and WR 38a based on HST PC images with superb angular resolution. We identify which stars are associated with the WR pair based on their derived reddening and location in a color-magnitude diagram. Our results are mainly consistent with those of Shorlin et al. (2004), but we 


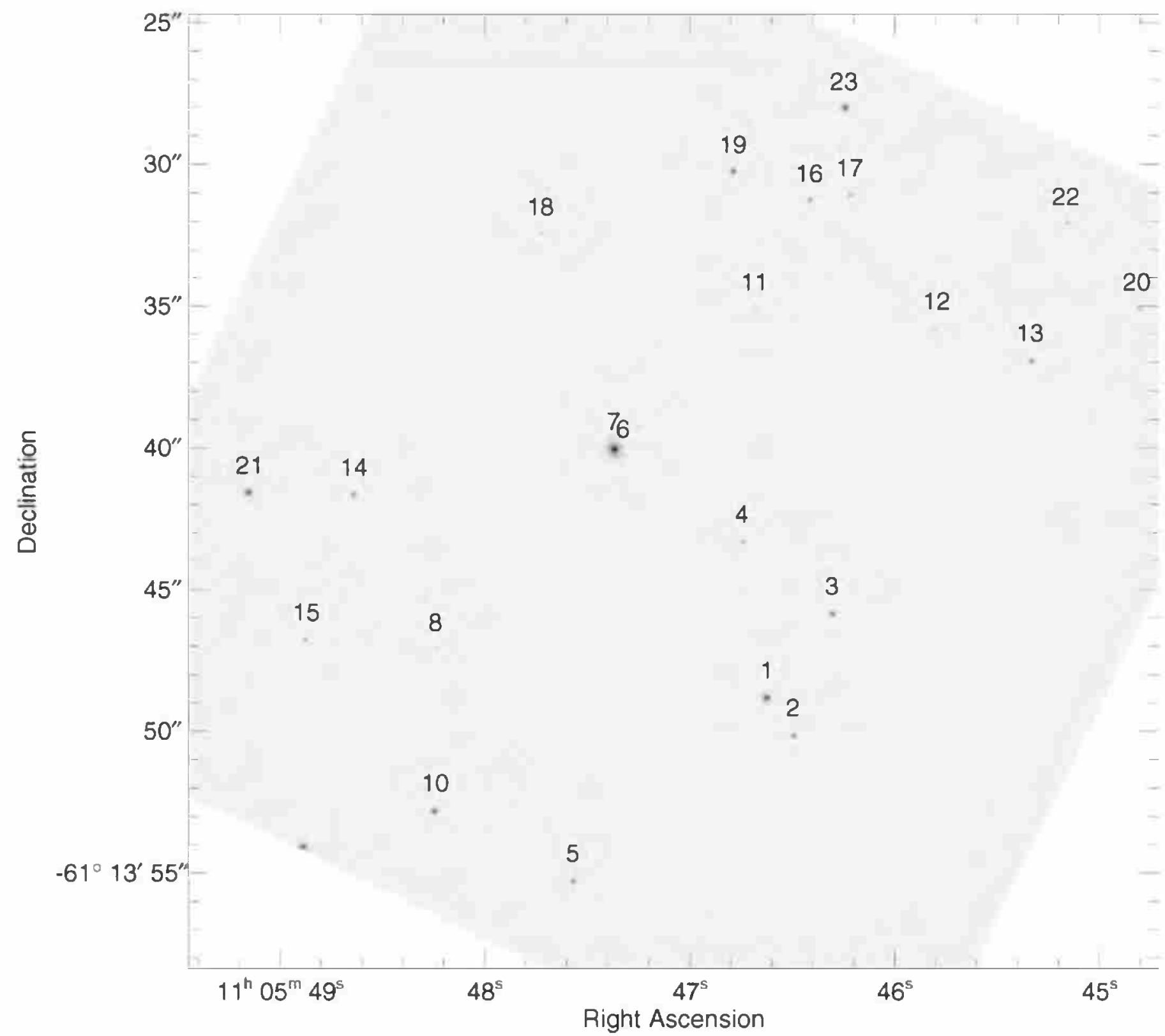

Fig. 1. - HST PC image of WR 38 (star 1) and WR 38a (star 21) made with the F555W filter (placed according to J2000.0 coordinates). The identification number above each star corresponds to the system used in Tables 1 and 2 .

argue that the acceptable distance range is such that the WR pair and its surrounding cluster may reside in the distant portion of the Carina spiral arm.

\section{OBSERVATIONS AND DATA REDUCTION}

Observations of the fields of WR 38 and WR 38 a were obtained in 1996 March (JD 2,450,166.58) through the F336W, F439W, and F555W filters as part of our HST WFPC2 survey of Galactic WR stars. These WFPC2 filters were designed to correspond to the Johnson $U B V$ filter set (Johnson \& Morgan 1953; Johnson et al. 1966), but because of the strong emission lines present in WR spectra the transformation from the HST to the Johnson system is approximate at best for them. In addition, the WFPC2 F336W filter suffers from a red leak that may cause dusty WR or other very red stars to appear brighter than in a similar $U$-band image. Therefore, all the data reductions and analyses were performed within the WFPC2 synthetic magnitude system (Holtzman et al. 1995).

We centered the WR stars within the PC chip to take advantage of the high resolution (scale of $0^{\prime \prime} 046$ pixel $^{-1}$ ). The observations also utilized a high gain setting, with a threshold of $\sim 53,000$ photoelectrons pixel ${ }^{-1}$, to optimize the discovery of faint companions. For each observation we made two exposures to facilitate the removal of cosmic rays $(40 \mathrm{~s}$ for the $\mathrm{F} 336 \mathrm{~W}, 10 \mathrm{~s}$ for the $\mathrm{F} 439 \mathrm{~W}$, and $2 \mathrm{~s}$ for the $\mathrm{F} 555 \mathrm{~W}$ filter). Since these were SNAPSHOT-type observations, which are designed to fill holes in the $H S T$ schedule, the observations were taken using only one guide star. Fortunately, the short exposure times resulted in no discernible loss of data quality due to telescope motion, although this did place limits on the accuracy of the absolute astrometry, as the telescope may have rolled during the observations (Baggett 2002; Gonzaga 2002; Nelan \& Makidon 2002). 
WR 38 is sufficiently close to WR 38 a that both stars could be captured within the same WFPC2 image (Fig. 1). The HST image clearly shows a number of components surrounding both WR stars that are not fully resolved in ground-based images (Shara et al. 1991; Shorlin et al. 2004). Without spectroscopic data we can only estimate the identity of the WR star within each stellar grouping by assuming it is the brightest object within the existing astrometric error box. The numbering system we adopted is based on angular separation from WR 38 (star 1), where WR $38 \mathrm{a}$ is labeled as star 21.

After normal pipeline processing (Baggett 2002), we aligned the six raw images using the task IMALIGN in IRAF/STSDAS. ${ }^{4}$ We then combined each pair of images per filter using the routine CRREJ. We identified the stars in each combined image using the task DAOFIND with the detection threshold set at $10 \sigma$ over the background level. We determined a background value by using the IMEXAMINE routine to derive the mean background value within $5 \times 5$ pixel boxes located at $5-10$ well-distributed positions across the image. The single average of these values was used for the detection threshold.

Because the observations were made with just one guide star, the images may suffer from telescope roll and jitter. Consequently, the point-spread function (PSF) of the stellar images may be asymmetrical, leading the DAOFIND algorithm to select false stars. Thus, all the detected stars were visually inspected before acceptance.

We used this list as input to the IRAF/STSDAS package METRIC to derive the astrometric information given in Table 1. METRIC translates the WFPC2 pixel positions to celestial coordinates after correcting for geometric distortion. The relative positions of well-exposed stars are accurate to better than $0^{\prime \prime} .005$ for targets imaged on one chip (errors are primarily due to centering uncertainties). However, while the relative positions are highly accurate, the absolute positions of the objects are subject to larger errors of $\pm\left(0.5-5^{\prime \prime}\right)$ due to the use of a single guide star (Baggett 2002; Gonzaga 2002; Nelan \& Makidon 2002). Fifteen of the stars in Table 1 appear in the 2MASS All-Sky Catalog of Point Sources (Cutri et al. 2003), and the mean differences in the coordinates are $\alpha$ (2MASS) $-\alpha(H S T)=+0.175 \pm 0.008 \mathrm{~s}$ and $\delta(2 \mathrm{MASS})-\delta(H S T)=+0^{\prime \prime} 14 \pm 0^{\prime \prime} 18$.

We performed aperture photometry using the IRAF package DAOPHOT (Stetson 1987). We chose aperture photometry over PSF photometry because of the difficulties involved in fitting the PSF due to the small telescope motions. We used an 11 pixel $(0$ ".5) radius aperture to determine the total stellar flux, and we estimated the sky background using the OFILTER algorithm within the PHOT package of DAOPHOT (as recommended by Ferguson 1996).

A number of photometric corrections are required to transform from raw to calibrated magnitudes (Baggett 2002). The stellar magnitudes were corrected for charge transfer efficiency using the Whitmore et al. (1999) formula for faint sources and the Dolphin $(2000)^{5}$ formula for more luminous sources (brighter than $100 e^{-}$). Geometric distortion effects were corrected using a scheme that rescaled the flux measurements of the stars based on their positions within the image. Contamination corrections were made using information taken from the WFPC2 Web site. ${ }^{6}$

\footnotetext{
${ }^{4}$ IRAF is distributed by the National Optical Astronomy Observatory, which is operated by AURA, Inc., under cooperative agreement with the National Science Foundation.

${ }^{5}$ See http://www.noao.edu/staff/dolphin/wfpc2_calib.

${ }^{6}$ See http://www.stsci.edu/instruments/wfpc2.
}

TABLE 1

Astrometric Data for the WR 38/WR 38a Cluster

\begin{tabular}{|c|c|c|c|c|}
\hline Field Number & $\alpha(\mathrm{J} 2000.0)$ & $\delta(\mathrm{J} 2000.0)$ & $\begin{array}{l}\text { Separation }{ }^{\mathrm{a}} \\
\text { (arcsec) }\end{array}$ & $\begin{array}{l}\text { P.A. }^{a} \\
\text { (deg) }\end{array}$ \\
\hline $1 \ldots$ & 110546.62 & -611348.8 & & \\
\hline 2 & 110546.49 & -611350.2 & 1.63 & 215.2 \\
\hline 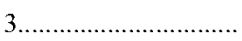 & 110546.30 & -611345.9 & 3.75 & 322.1 \\
\hline 4 & 110546.74 & -611343.3 & 5.57 & 8.9 \\
\hline 5................................ & 110547.56 & -611355.3 & 9.38 & 133.4 \\
\hline 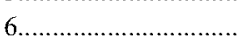 & 110547.32 & -611340.3 & 9.90 & 30.7 \\
\hline 7 & 110547.36 & -611340.1 & 10.28 & 31.5 \\
\hline 8 & 110548.23 & $\begin{array}{lll}-61 & 13 & 47.1\end{array}$ & 11.79 & 81.7 \\
\hline 9 & 110546.81 & -611400.0 & 11.87 & 173.2 \\
\hline 10 & 110548.24 & -611352.8 & 12.34 & 108.8 \\
\hline $11 \ldots$ & 110546.68 & -611335.1 & 13.69 & 1.8 \\
\hline $12 \ldots \ldots \ldots \ldots \ldots \ldots \ldots \ldots \ldots$ & 110545.79 & -611335.8 & 14.32 & 335.3 \\
\hline $13 \ldots \ldots \ldots$ & 110545.33 & -611336.9 & 15.08 & 322.0 \\
\hline 14 & 110548.63 & -611341.7 & 16.21 & 63.8 \\
\hline 15 & 110548.87 & -611346.8 & 16.34 & 82.8 \\
\hline 16 & 110546.41 & -611331.3 & 17.59 & 355.1 \\
\hline 17 & 110546.21 & -611331.1 & 17.95 & 350.6 \\
\hline $18 \ldots \ldots$ & 110547.72 & -611332.5 & 18.17 & 26.0 \\
\hline $19 \ldots$ & 110546.78 & -611330.3 & 18.58 & 3.6 \\
\hline $20 \ldots \ldots$ & 110544.81 & -611335.1 & 18.91 & 316.4 \\
\hline $21^{\mathrm{b}} \ldots$ & 110549.14 & -611341.6 & 19.59 & 68.4 \\
\hline $22 \ldots$ & 110545.16 & -611332.1 & 19.76 & 327.7 \\
\hline $23 \ldots \ldots \ldots \ldots \ldots \ldots \ldots$ & 110546.24 & -611328.1 & 20.93 & 352.5 \\
\hline 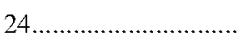 & 110544.49 & -611334.1 & 21.31 & 313.8 \\
\hline 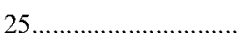 & 110549.76 & -611348.5 & 22.65 & 89.2 \\
\hline
\end{tabular}

NOTEs.- Units of right ascension are hours, minutes, and seconds, and units of declination are degrees, arcminutes, and arcseconds. To convert to the 2MASS frame, add $0.17 \mathrm{~s}$ to $\alpha$ and $0^{\prime \prime} 1$ to $\delta$.

${ }^{\text {a }}$ With respect to WR 38 (target 1).

${ }^{\mathrm{b}}$ WR 38a.

The 34th row effect was corrected using the scheme of Anderson \& King (1999). Most significantly, the zero-point corrections for the F555W and F439W magnitude data were calculated using the Dolphin (2000) updates to the original Holtzman et al. (1995) offsets. The Dolphin (2000) revisions to the Holtzman et al. (1995) zero points $(\approx 0.01 \mathrm{mag})$ are not the result of temporal effects but rather of improved calibration methods, and hence, they have greater accuracy. Unfortunately, Dolphin did not provide updates for the F336W filter, so we used the Holtzman et al. (1995) calibration for the F336W filter images.

Our final magnitudes in the HST system are collected in Table 2. We have included a transformation of the $H S T$ wide-band magnitudes to Johnson $B V$ using the scheme of Dolphin (2000). We caution that this scheme may not be reliable for the WR stars, since both the $B$ and $V$ bandpasses are sensitive to the strong emission lines present in WR spectra (van Genderen et al. 1987). We did not attempt to transform our F336W magnitudes to Johnson $U$, since the latter straddles the Balmer jump, while the former is sensitive mainly to shorter wavelength flux. We have also included in Table 2 the corresponding identification number (STP) and magnitude information from the work of Shorlin et al. (2004) for seven stars in common (mainly cluster members).

We found that there are systematic differences between our transformed $V$ and $B-V$ magnitudes and those of Shorlin et al. (2004). We omitted the WR stars from this comparison because of our concerns about the transformation to Johnson magnitudes, and this left five stars in common between our samples. We find mean differences of $\left\langle V_{H S T}-V_{\mathrm{STP}}\right\rangle=0.24 \pm 0.03$ and $\left\langle(B-V)_{H S T}-(B-V)_{\mathrm{STP}}\right\rangle=-0.12 \pm 0.09$, i.e., the mean 
TABLE 2

Uncorrected Photometric Data for the WR 38/WR 38a Cluster

\begin{tabular}{|c|c|c|c|c|c|}
\hline Star & $m_{\mathrm{F} 555 \mathrm{~W}}$ & $m_{\mathrm{F} 439 \mathrm{~W}}-m_{\mathrm{F} 555 \mathrm{~W}}$ & $m_{\mathrm{F} 336 \mathrm{~W}}-m_{\mathrm{F} 439 \mathrm{~W}}$ & $V$ & $B-V$ \\
\hline $1^{\mathrm{a}} \ldots \ldots \ldots$ & $15.00 \pm 0.03$ & $1.21 \pm 0.05$ & $0.37 \pm 0.06$ & $14.97 \pm 0.03$ & $1.13 \pm 0.05$ \\
\hline STP $40 \ldots \ldots \ldots \ldots \ldots \ldots \ldots$ & $\ldots$ & $\ldots$ & $\ldots$ & 14.66 & 1.28 \\
\hline 2 & $16.47 \pm 0.05$ & $1.21 \pm 0.13$ & $-0.18 \pm 0.14$ & $16.44 \pm 0.06$ & $1.13 \pm 0.13$ \\
\hline 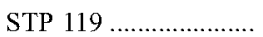 & $\ldots$ & $\ldots$ & $\ldots$ & 16.21 & 1.23 \\
\hline З & $16.19 \pm 0.05$ & $1.09 \pm 0.10$ & $0.02 \pm 0.11$ & $16.16 \pm 0.05$ & $1.03 \pm 0.10$ \\
\hline 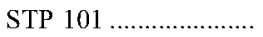 & $\ldots$ & $\ldots$ & $\ldots$ & 15.90 & 1.27 \\
\hline 4 & $17.60 \pm 0.12$ & $1.35 \pm 0.42$ & $\ldots$ & $17.57 \pm 0.12$ & $1.24 \pm 0.42$ \\
\hline 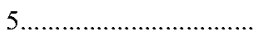 & $16.81 \pm 0.06$ & $1.32 \pm 0.19$ & $0.13 \pm 0.24$ & $16.79 \pm 0.07$ & $1.21 \pm 0.19$ \\
\hline STP $138 \ldots \ldots \ldots \ldots \ldots \ldots \ldots \ldots \ldots \ldots$ & $\ldots$ & $\ldots$ & $\ldots$ & 16.56 & 1.33 \\
\hline 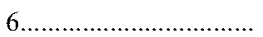 & $17.39 \pm 0.12$ & $\ldots$ & $\ldots$ & $\ldots$ & $\ldots$ \\
\hline $7 \ldots \ldots \ldots$ & $13.27 \pm 0.03$ & $1.41 \pm 0.04$ & $0.20 \pm 0.04$ & $13.25 \pm 0.03$ & $1.29 \pm 0.05$ \\
\hline $8 \ldots \ldots \ldots \ldots \ldots$ & $18.79 \pm 0.39$ & $\ldots$ & $\ldots$ & $\ldots$ & $\ldots$ \\
\hline 9 & $18.70 \pm 0.40$ & $\ldots$ & $\ldots$ & $\ldots$ & $\ldots$ \\
\hline 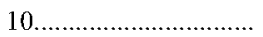 & $15.83 \pm 0.04$ & $1.21 \pm 0.09$ & $0.09 \pm 0.10$ & $15.80 \pm 0.04$ & $1.13 \pm 0.09$ \\
\hline STP $76 \ldots \ldots \ldots \ldots \ldots . . . . . . . . . . .$. & $\ldots$ & $\ldots$ & $\ldots$ & 15.51 & 1.28 \\
\hline $11 \ldots \ldots \ldots \ldots \ldots \ldots \ldots$ & $18.63 \pm 0.32$ & $\ldots$ & $\ldots$ & $\ldots$ & $\ldots$ \\
\hline 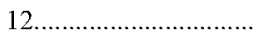 & $18.69 \pm 0.35$ & $\ldots$ & $\ldots$ & $\ldots$ & $\ldots$ \\
\hline 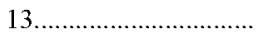 & $16.96 \pm 0.07$ & $0.85 \pm 0.16$ & $0.28 \pm 0.20$ & $16.94 \pm 0.08$ & $0.82 \pm 0.16$ \\
\hline $14 \ldots \ldots+\ldots+\cdots$ & $16.74 \pm 0.07$ & $1.42 \pm 0.22$ & $0.08 \pm 0.26$ & $16.72 \pm 0.07$ & $1.30 \pm 0.22$ \\
\hline STP $137 \ldots \ldots \ldots \ldots \ldots \ldots$ & $\ldots$ & $\ldots$ & $\ldots$ & 16.51 & 1.30 \\
\hline $15 \ldots \ldots \ldots \ldots \ldots \ldots \ldots \ldots$ & $17.55 \pm 0.13$ & $1.41 \pm 0.43$ & $\ldots$ & $17.53 \pm 0.13$ & $1.29 \pm 0.43$ \\
\hline . & $17.11 \pm 0.09$ & $1.24 \pm 0.25$ & $0.00 \pm 0.29$ & $17.09 \pm 0.09$ & $1.15 \pm 0.26$ \\
\hline $17 \ldots \ldots \ldots \ldots \ldots \ldots \ldots \ldots \ldots \ldots \ldots \ldots \ldots \ldots \ldots \ldots$ & $17.83 \pm 0.16$ & $1.22 \pm 0.47$ & $\ldots$ & $17.80 \pm 0.16$ & $1.13 \pm 0.47$ \\
\hline 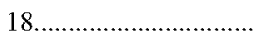 & $18.79 \pm 0.40$ & $\ldots$ & $\ldots$ & $\ldots$ & $\ldots$ \\
\hline 19 & $16.00 \pm 0.04$ & $1.07 \pm 0.09$ & $-0.04 \pm 0.10$ & $15.98 \pm 0.05$ & $1.01 \pm 0.09$ \\
\hline 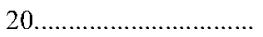 & $17.99 \pm 0.17$ & $0.83 \pm 0.39$ & $\ldots$ & $17.97 \pm 0.17$ & $0.80 \pm 0.39$ \\
\hline 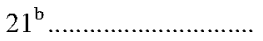 & $15.33 \pm 0.04$ & $1.24 \pm 0.07$ & $-0.13 \pm 0.08$ & $15.30 \pm 0.04$ & $1.15 \pm 0.07$ \\
\hline 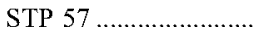 & $\ldots$ & $\ldots$ & $\ldots$ & 15.12 & 1.15 \\
\hline $22 \ldots \ldots \ldots \ldots \ldots \ldots \ldots \ldots \ldots \ldots \ldots \ldots \ldots \ldots \ldots \ldots$ & $18.12 \pm 0.21$ & $\ldots$ & $\ldots$ & $\ldots$ & $\ldots$ \\
\hline $23 \ldots \ldots \ldots \ldots \ldots \ldots \ldots \ldots \ldots \ldots \ldots \ldots \ldots \ldots \ldots \ldots \ldots$ & $15.38 \pm 0.04$ & $0.45 \pm 0.05$ & $0.42 \pm 0.05$ & $15.36 \pm 0.04$ & $0.45 \pm 0.06$ \\
\hline . & $18.22 \pm 0.23$ & $\ldots$ & $\ldots$ & $\ldots$ & $\ldots$ \\
\hline . & $17.50 \pm 0.13$ & $1.23 \pm 0.38$ & $\ldots$ & $17.47 \pm 0.13$ & $1.15 \pm 0.38$ \\
\hline
\end{tabular}

NoтE.-The corresponding stars and results from Shorlin et al. (2004) (e.g., STP 40) are listed below their HST values. ${ }^{\text {a }}$ WR 38.

${ }^{\mathrm{b}}$ WR 38a.

differences are larger than the standard deviations from the mean differences (and the standard deviations are comparable to the expected observational errors). These differences are probably due to errors in the adopted zero points for the WFPC2 images. We have found, for example, that there is an intrinsic scatter of order $0.1 \mathrm{mag}$ between the observed and synthetic $H S T$ magnitudes for some two dozen WR stars with near-UV spectroscopy from the International Ultraviolet Explorer satellite and optical spectrophotometry (Torres \& Massey 1987; Torres-Dodgen \& Massey 1988). While part of this scatter may be due to errors in the calibration of the spectrophotometry, we caution that zero-point offsets of order $0.1 \mathrm{mag}$ may be required to place our photometric results on an absolute scale. Thus, the relative magnitudes for stars within a given image are secure, but color indices formed from magnitude differences between images may require a zero-point shift. In $\S 3$ we describe one way of estimating the zero-point adjustments based on a reddening analysis of multiwavelength photometry.

\section{INTERSTELLAR REDDENING}

Extinction due to interstellar dust decreases at longer wavelengths, so the best approach to determining reddening and extinction is to compare both long- and short-wavelength flux measurements with spectral models transformed using a suitable reddening law. We were pleased to learn that 15 of the 25 targets in our $H S T$ field are included in the 2MASS All-Sky Catalog of Point Sources (Cutri et al. 2003), which lists $J, H$, and $K$ magnitudes for most of them. Here we examine the 2MASS, HST, and, where available, Johnson magnitudes from Shorlin et al. (2004) in order to estimate the reddening for each target. Our approach is to calculate synthetic magnitudes in each band based on a consistent spectral flux distribution for a given zero-age main-sequence (ZAMS) stellar effective temperature, $T_{\text {eff, }}$ and a given reddening, $E(B-V)$.

We assembled a small grid of theoretical spectra covering the wavelength range of interest for ZAMS stars along the upper main sequence. We used model spectra based on nonLTE, line-blanketed model atmospheres from Lanz \& Hubeny (2003) for the O stars (for effective temperatures of 27,500, 35,000 , and $45,000 \mathrm{~K}$ ), and we adopted spectra from LTE models by R. L. Kurucz for the B stars (for 10,000, 15,000, 20,000, and $24,000 \mathrm{~K}$ ). The gravity $\log g$ for each temperature was taken from the 1 Myr ZAMS sequence of Lejeune \& Schaerer (2001). In addition to the computational differences between the Kurucz and Lanz \& Hubeny models, the former assumes a microturbulence of $4 \mathrm{~km} \mathrm{~s}^{-1}$, while the latter uses $10 \mathrm{~km} \mathrm{~s}^{-1}$. Despite these differences, the predicted flux distributions generally make a smooth transition at the temperature boundary

\footnotetext{
${ }^{7}$ See http://kurucz.harvard.edu/grids/gridP00/fp00k4.pck.
} 
TABLE 3

ZAMS Intrinsic Colors from Synthetic Magnitudes

\begin{tabular}{|c|c|c|c|c|c|c|c|c|c|}
\hline $\begin{array}{c}T_{\text {eff }} \\
(\mathrm{K})\end{array}$ & $m(336)-V$ & $m(439)-V$ & $m(555)-V$ & $U-V$ & $B-V$ & $J-V$ & $H-V$ & $K-V$ & $K_{0}$ \\
\hline $10,000 \ldots \ldots \ldots \ldots$ & -0.15 & -0.02 & -0.00 & -0.07 & -0.02 & 0.06 & 0.08 & 0.08 & 1.46 \\
\hline $15,000 \ldots \ldots \ldots \ldots \ldots$ & -1.04 & -0.14 & -0.01 & -0.68 & -0.14 & 0.34 & 0.39 & 0.44 & 0.46 \\
\hline $20,000 \ldots \ldots \ldots \ldots \ldots$ & -1.42 & -0.20 & -0.02 & -0.95 & -0.19 & 0.49 & 0.57 & 0.63 & -0.44 \\
\hline $24,000 \ldots \ldots \ldots \ldots \ldots$ & -1.63 & -0.22 & -0.02 & -1.10 & -0.22 & 0.58 & 0.67 & 0.76 & -1.09 \\
\hline $27,500 \ldots \ldots \ldots \ldots \ldots \ldots$ & -1.75 & -0.26 & -0.02 & -1.20 & -0.25 & 0.63 & 0.72 & 0.82 & -1.66 \\
\hline $35,000 \ldots \ldots \ldots \ldots \ldots$ & -1.90 & -0.28 & -0.02 & -1.32 & -0.27 & 0.70 & 0.81 & 0.92 & -2.97 \\
\hline $45,000 \ldots \ldots \ldots \ldots \ldots$ & -1.96 & -0.28 & -0.02 & -1.36 & -0.28 & 0.71 & 0.82 & 0.93 & -4.79 \\
\hline
\end{tabular}

between them (where non-LTE effects for hydrogen become negligible).

We calculated synthetic magnitudes for these spectra using the SYNPHOT routine CALCPHOT (Bushouse \& Simon 1998), which is distributed with the STSDAS package in IRAF. The software includes the spectral response functions for the HST wide-band filters, as well as those for the standard Johnson filters. However, we used the response functions from Cohen et al. (2003) to simulate the sampling of the spectra in the IR with the 2MASS filter system. The procedure returns synthetic magnitudes on the VEGAMAG system, so defined that Vega has a magnitude 0.0 in all filters. Since Vega actually has magnitudes $U=0.02, B=0.02$, and $V=0.03$ in the Johnson system, we adjusted both the Johnson and corresponding HST synthetic magnitudes by these amounts to make them consistent with observations. The resulting synthetic ZAMS colors relative to Johnson $V$ and the absolute $K$ magnitude are collected in Table 3 for our grid of stellar effective temperatures. These are generally in reasonable agreement with Johnson color indices published by Wegner (1994) and Bessell et al. (1998), except perhaps for the shortest wavelengths and hottest stars. For example, Turner (1976) and Underhill et al. (1979) argue that the hottest $O$ stars have $B-V=-0.32$, versus our lower limit of $B-V=-0.28$. Note that the $U-V$ and $B-V$ color indices are often based on uncertain dereddening procedures for O-type stars, which may tend to produce colors that are too blue. The lowest $B-V=$

-0.28 for the $45,000 \mathrm{~K}$ model is consistent with that for the bluest $O$ stars in the Galaxy (Maiz-Apellániz et al. 2004) and in the LMC (Fitzpatrick 1988).

We next transformed each of these model spectra for a grid of 40 values of $E(B-V)$ from 0 to 3.9 using the reddening scheme of Fitzpatrick (1999) for a ratio of total to selective extinction of $R=3.1$ (the same as adopted by Shorlin et al. 2004). We then used CALCPHOT to determine the predicted color indices as a function of the reddening. A set of sample reddening curves is shown in Figure 2 for the 35,000 K model spectrum. Note that unlike for the other filters, the color index trend for the $H S T$ F336W filter undergoes a reversal at large reddening because of a red leak in this filter.

We performed a numerical grid search to find the stellar temperature and reddening that best matched a given set of IR and optical color indices. We found this scheme was able to confirm the known reddening in a number of test cases for OB stars for which Johnson $U, B$, and $V$ and 2MASS $J, H$, and $K$ magnitudes were available, but the method is not applicable to stars with temperatures cooler than our grid limit of $10,000 \mathrm{~K}$. We applied the method to all the stars in our WFPC2 field for which 2MASS photometric data existed. These stars are listed in Table 4, which gives our assigned number, the 2MASS designation, the number adopted by Shorlin et al. (2004), our derived reddening, and dereddened estimates of $m(336)-K$ and $K$ (see below). There are several stars with relatively low reddening that are probably foreground objects, but most of the other stars have a reddening in the range $E(B-V)=1.5-2.0$ (with the possible exception of the faint star 8 , which may be a foreground cool star). There are four cluster stars with a complete set of WFPC2, Johnson (Shorlin et al. 2004), and 2MASS magnitudes (after omission of the two WR stars for which the method may be poor), and the mean reddening for these four stars is $E(B-V)=1.63 \pm 0.05$ (mean

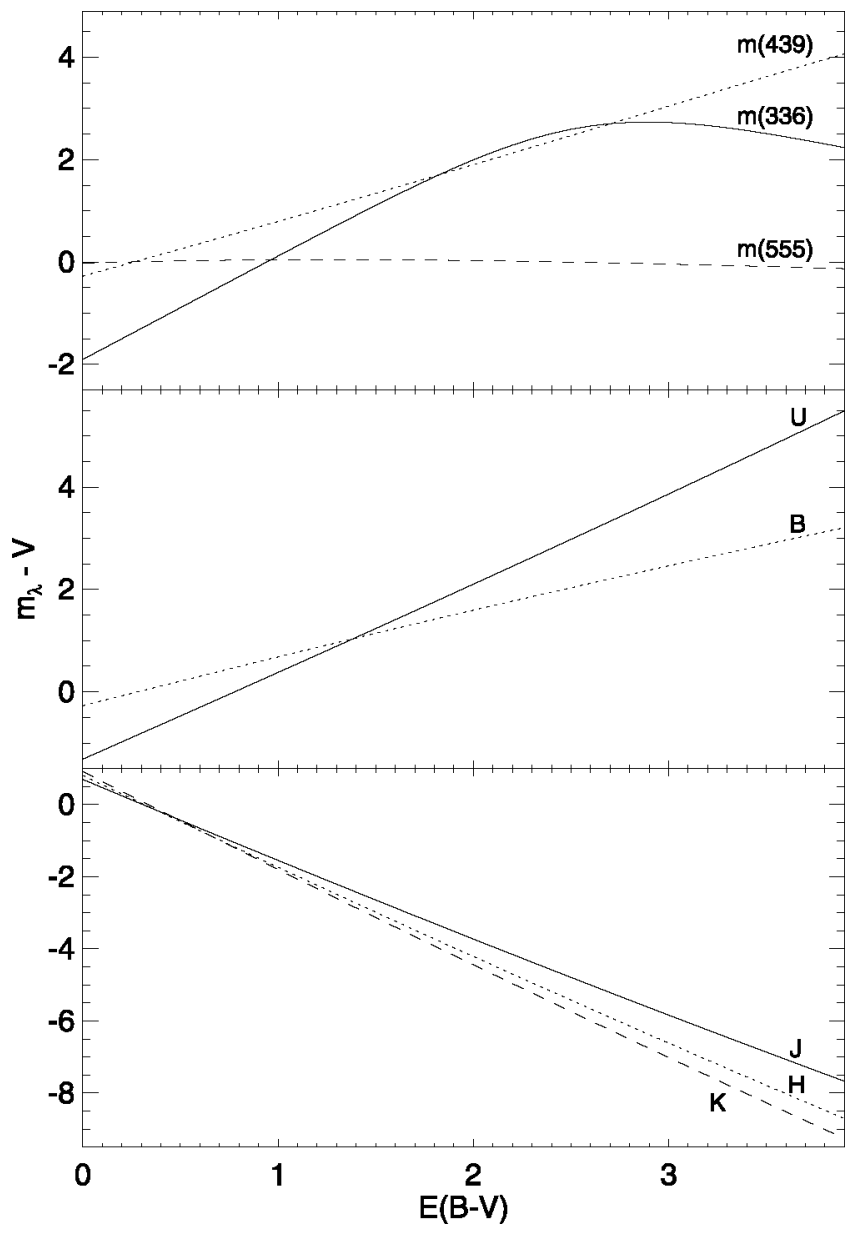

FIG. 2.-Reddening curves based on synthetic magnitudes from SYNPHOT/ CALCPHOT for a spectrum from a model with $T_{\text {eff }}=35,000 \mathrm{~K}$ and $\log g=$ 4.16 (Lanz \& Hubeny 2003) and for a reddening law with $R=3.1$ (Fitzpatrick 1999). The top panel shows how the $H S T$ color indices $m(336)-V, m(439)-V$, and $m(555)-V$ vary with reddening $E(B-V)$. The $m(336)-V$ trend reverses at large reddening because of the red leak in the $\mathrm{F} 336 \mathrm{~W}$ filter. The middle panel shows the variations for the Johnson $U-V$ and $B-V$ indices, while the bottom panel shows the same for the IR indices, $J-V, H-V$, and $K-V$. 
TABLE 4

$R=3.1$ Reddentng Parameters for Stars with 2MASS Photometry

\begin{tabular}{|c|c|c|c|c|c|c|}
\hline Field Number & 2MASS ID & STP ID & $E(B-V)$ & {$[m(336)-K]-E(m(336)-K)$} & $K-A(K)$ & Comment \\
\hline$\ldots \ldots \ldots \ldots . .$. & $11054642-6113487$ & 40 & $1.46 \pm 0.12$ & $-1.35 \pm 0.06$ & $10.43 \pm 0.05$ & WR 38 \\
\hline $3 \ldots \ldots \ldots \ldots \ldots \ldots \ldots \ldots \ldots \ldots \ldots \ldots \ldots$ & $11054613-6113457$ & 101 & $1.51 \pm 0.09$ & $-2.68 \pm 0.10$ & $12.16 \pm 0.07$ & All bands \\
\hline 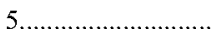 & $11054738-6113550$ & 138 & $1.70 \pm 0.09$ & $-2.81 \pm 0.17$ & $12.31 \pm 0.03$ & All bands \\
\hline $7 \ldots \ldots \ldots \ldots \ldots \ldots \ldots \ldots \ldots \ldots \ldots \ldots \ldots$ & $11054719-6113398$ & $\ldots$ & $1.84 \pm 0.11$ & $-2.91 \pm 0.03$ & $8.36 \pm 0.02$ & $\ldots$ \\
\hline $8 \ldots \ldots \ldots \ldots \ldots \ldots \ldots \ldots \ldots \ldots \ldots \ldots \ldots \ldots$ & $11054805-6113469$ & $\ldots$ & $2.51 \pm 0.24$ & $\ldots$ & $11.97 \pm 0.06$ & Foreground? \\
\hline $9 \ldots$ & $11054664-6114003$ & $\ldots$ & $1.70 \pm 0.20$ & $\ldots$ & $14.24 \pm 0.12$ & \\
\hline $10 \ldots \ldots \ldots \ldots \ldots \ldots \ldots \ldots$ & $11054806-6113526$ & 76 & $1.61 \pm 0.10$ & $-2.71 \pm 0.09$ & $11.54 \pm 0.06$ & All bands \\
\hline $12 \ldots \ldots \ldots \ldots \ldots \ldots$ & $11054562-6113358$ & $\ldots$ & $0.20 \pm 0.10$ & $\ldots$ & $\ldots$ & Foreground \\
\hline .................. & $11054515-6113367$ & $\ldots$ & $0.70 \pm 0.08$ & $\ldots$ & $\ldots$ & Foreground \\
\hline $14 \ldots \ldots \ldots \ldots \ldots \ldots \ldots$ & $11054845-6113414$ & 137 & $1.69 \pm 0.15$ & $-2.62 \pm 0.17$ & $12.16 \pm 0.08$ & All bands but $J$ \\
\hline $15 \ldots \ldots \ldots \ldots \ldots \ldots \ldots$ & $11054869-6113465$ & $\ldots$ & $1.47 \pm 0.06$ & $\ldots$ & $13.21 \pm 0.09$ & $\ldots$ \\
\hline $19 \ldots \ldots \ldots \ldots \ldots \ldots \ldots \ldots$ & $11054659-6113301$ & $\ldots$ & $1.52 \pm 0.10$ & $-2.98 \pm 0.09$ & $12.13 \pm 0.06$ & $\ldots$ \\
\hline $21 \ldots \ldots \ldots \ldots \ldots \ldots \ldots$ & $11054896-6113413$ & 57 & $1.49 \pm 0.09$ & $-1.60 \pm 0.06$ & $10.33 \pm 0.04$ & WR 38a \\
\hline $23 \ldots \ldots \ldots \ldots \ldots \ldots$ & $11054607-6113279$ & $\ldots$ & $0.55 \pm 0.04$ & $-0.56 \pm 0.12$ & $13.84 \pm 0.11$ & Foreground \\
\hline $25 \ldots \ldots \ldots \ldots \ldots \ldots \ldots$ & $11054956-6113482$ & $\ldots$ & $1.39 \pm 0.09$ & $\ldots$ & $13.28 \pm 0.06$ & $\ldots$ \\
\hline
\end{tabular}

error), in good agreement with the result of $E(B-V)=1.60 \pm$ 0.02 from Shorlin et al. (2004).

We can use these reddening fits to return to the question of magnitude zero-point offsets that we raised in $\S 2$. Table 5 lists the average values of the residuals from the fit for each filter based on the same sample of four stars with complete magnitude coverage. These represent any systematic offsets between the observed magnitudes and the synthetic magnitudes derived from the theoretical spectra, so they encompass any lingering problems in the observational zero points, filter responses, and wavelength-specific deficiencies in the theoretical models. The offsets in Table 5 are generally small and comparable to the scatter in the residuals, but there are some interesting exceptions. We see, for example, that the mean difference in the residuals for $m(555)-V$ is $0.21 \pm 0.04$, which is the same within errors as the difference between the transformed $V_{H S T}$ and $V_{\text {STP }}$ that we found in $\S 2$. Similarly, the residuals in $m(439)-m(555)$ suggest that our uncorrected color index is too blue in the same way as demonstrated in our comparison of our transformed $B-V$ with the colors from Shorlin et al. (2004). Thus, the mean residuals in Table 5 lead us to revise our original WFPC2 zero points to 18.305 for the F336W filter, 20.036 for the F439W filter, and 21.496 for the F555W filter. An application of these zero-point corrections should provide consistent results across the entire set of photometric measurements. We give in the fifth and sixth columns of Table 4 the individually dereddened color index $m(336)-K$ (including a zero-point offset of $-0.35 \pm 0.09$ from the mean residuals in Table 5) and extinction-corrected $K$ (using a zero-point offset of $+0.15 \pm 0.07$ from Table 5) that we use in $\S 4$ to estimate the cluster distance.

TABLE 5

Average Magnitude Residuals from Reddening Fits

\begin{tabular}{|c|c|}
\hline Filter & $\left\langle m_{\lambda}\right.$ (observed) $-m_{\lambda}$ (synthetic) $\rangle$ \\
\hline F336W.................... & $0.20 \pm 0.05$ \\
\hline F439W .................... & $0.04 \pm 0.04$ \\
\hline F555W ....................... & $0.23 \pm 0.04$ \\
\hline 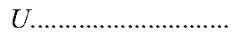 & $-0.17 \pm 0.06$ \\
\hline$B \ldots \ldots \ldots \ldots \ldots \ldots \ldots \ldots \ldots$ & $0.03 \pm 0.06$ \\
\hline ................ & $0.02 \pm 0.01$ \\
\hline …............. & $0.00 \pm 0.01$ \\
\hline$H \ldots \ldots \ldots \ldots \ldots \ldots \ldots \ldots \ldots$ & $-0.06 \pm 0.09$ \\
\hline$K \ldots \ldots \ldots \ldots \ldots \ldots \ldots \ldots \ldots \ldots$ & $-0.15 \pm 0.07$ \\
\hline
\end{tabular}

We caution that these results are sensitive to the adopted value of the ratio of total to selective extinction $R$. This parameter is often estimated by comparing the reddening $E(B-V)$ with the extinction in the IR, and we used equations (A3)-(A5) from Fitzpatrick (1999) to estimate $R$ for the four stars with complete Johnson and 2MASS photometry. The mean derived value, $R=3.25 \pm 0.15$, is consistent within errors with our assumed value of $R=3.1$, but the application of reddening curves for $R=3.25$ leads to a mean reddening of $E(B-V)=1.51 \pm$ 0.06 for the same sample of four stars using the scheme outlined above. We discuss below how such a revision in the reddening law influences the distance estimate.

\section{DISTANCE TO THE CLUSTER}

We can estimate the distance to the cluster surrounding WR 38 and WR 38a from the positions of the stars in a dereddened colorextinction-corrected magnitude diagram. The spectral flux distributions of massive stars appear similar to the Rayleigh-Jeans tail of a blackbody spectrum in the optical and near-IR spectral range, and we need spectral line diagnostics ( primarily through spectral classification) in order to estimate the stellar effective temperature reliably (and thus establish the luminosity of the star relative to the main sequence). In the absence of spectral data, the best approach is to use a color index based on the widest wavelength range possible as a temperature parameter. We selected the $m(336)-K$ index for this purpose, which spans a range of nearly 3 mag over the OB star temperature range (compared with a range of only $\approx 0.26 \mathrm{mag}$ in $B-V$; see Table 3 ). We also decided to use $K$ for the magnitude ordinate in the colormagnitude diagram, since it is least affected by uncertainties in extinction.

The color-magnitude diagram for the WR 38 cluster in this magnitude system is illustrated in Figure 3. The $m(336)-K$ colors were dereddened and the $K$ magnitudes extinction-corrected using the individual $E(B-V)$ values determined for each star in $\S 3$ (Table 4). In addition, the zero-point corrections found from the mean residuals to the reddening solutions (Table 5) were applied to bring the observed magnitudes into the synthetic magnitude system needed to compare the observed and theoretical color-magnitude diagrams. There are five stars that have similar positions in Figure 3 that probably correspond to the cluster main sequence. One bright star, source 7 , is located above the main sequence, and it is either an evolved, luminous 


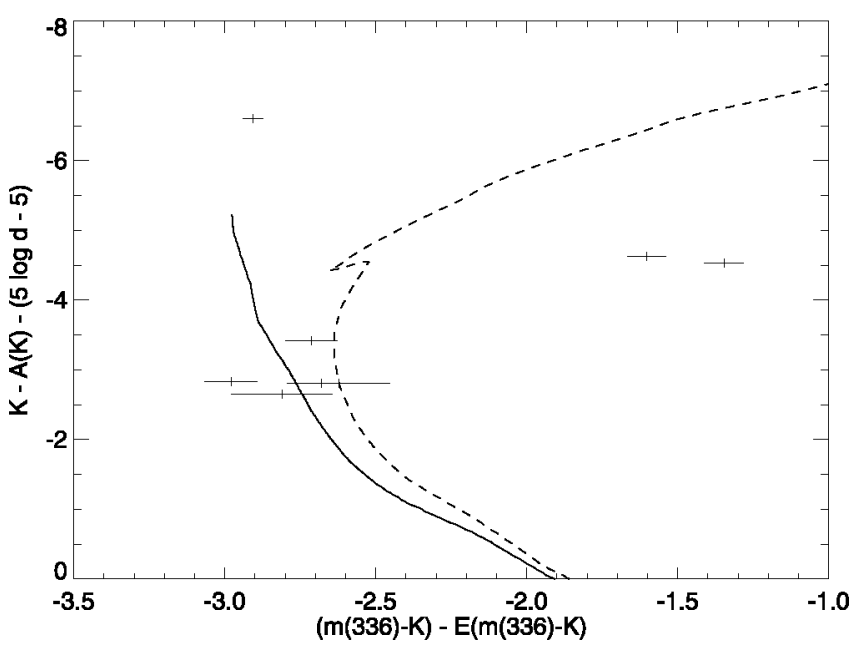

FIG. 3.-Color-magnitude diagram based on the dereddened color index $[m(336)-K]_{0}$ and the absolute $K$ magnitude (for a distance modulus of $5 \log d-$ $5=15.0$ ). The solid and dashed lines represent the 1 and $8 \mathrm{Myr}$ isochrones, respectively, from Lejeune \& Schaerer (2001), while the plus signs represent the magnitudes and colors of the probable cluster stars. Errors in the color index are represented by the horizontal lines through each symbol (errors in $K$ are comparable to the vertical symbol size). The two WR stars that appear in the right side of the diagram and the bright star (7) located at the top of the diagram were omitted in the main-sequence fitting procedure

star within the cluster or a foreground object. The two WR stars are found well to the right of the main sequence, which is probably due to the strong emission lines found in the $K$ band (Figer et al. 1997).

We adopted the 1 Myr ZAMS from the models of Lejeune \& Schaerer (2001) to determine the distance modulus from the difference of the observed and theoretical absolute magnitudes. We compared our synthetic color index $m(336)-K$ values from SYNPHOT/CALCPHOT for our temperature grid with the corresponding values from the tables of Lejeune \& Schaerer (2001), and we found a mean difference of $0.04 \pm 0.06 \mathrm{mag}$. We added this small offset to the model ZAMS color index from Lejeune \& Schaerer (2001) in order to compare the colors consistently in the synthetic system. We then compared the observed and model colors for a grid of assumed distance modulus values to determine the best-fit estimate. The derived distance modulus is $5 \log d-5=15.0_{-1.0}^{+1.5}$ based on the $\chi^{2}$ residuals of the fit (corresponding to a distance of $d=10_{-4}^{+12} \mathrm{kpc}$ ). At this distance, the five main-sequence stars in Figure 3 have colors and magnitudes of ZAMS stars in the temperature range 33,000-38,000 K, which is characteristic of O-type stars.

Our distance modulus estimate agrees within errors with that derived by Shorlin et al. (2004): $15.80 \pm 0.25$. The small difference in distance modulus between these investigations is probably due to differences in the adopted ZAMS isochrone. The ZAMS relation adopted by Shorlin et al. (2004) (from Turner 1976) is an empirical relation based on a somewhat evolved population of stars, and it is approximately $0.8 \mathrm{mag}$ brighter at $B-V=-0.30$ than the theoretical 1 Myr ZAMS that we adopted from Lejeune \& Schaerer (2001). Note that Schönberner \& Harmanec (1995) verified that the Geneva models used by Lejeune \& Schaerer (2001) yield absolute magnitudes that agree with those determined for eclipsing binaries after a correction is made for evolution from the ZAMS. We also show in Figure 3 the isochrone for an age of $8 \mathrm{Myr}$, approximately equal to the maximum age of clusters containing WR stars (Massey et al. 2001), and a comparison of the isochrones shows that we may be underestimating the distance modulus by $\approx 1$ mag if the cluster is significantly older than assumed. Unfortunately, we do not know the age of the cluster, and consequently the amount of evolutionary brightening at the upper end of the main sequence is unknown. Deeper photometric observations could resolve this problem through ZAMS fitting of less massive, unevolved stars.

If the ratio of total to selective extinction is revised upward to $R=3.25(\S 3)$, then the derived reddening is smaller, and the intrinsic $m(336)-K$ colors are larger than shown in Figure 3 . Such a revision would lead to a decrease in distance modulus to a value of $13.5 \mathrm{mag}$, slightly below the $1 \sigma$ error limit quoted above for the $R=3.1$ model fit. Thus, given the uncertainties in the cluster age and the reddening law, the range in acceptable distance modulus value may be somewhat larger than given above. Taken at face value, our analysis does not improve on the distance estimate from Shorlin et al. (2004), $14.5 \pm 1.6 \mathrm{kpc}$, but we caution that their error refers only to internal sources, and at this stage it is prudent to acknowledge an error range that accounts for systematic errors resulting from assumptions about the cluster isochrone and reddening law.

Our line of sight toward WR $38\left(l=290^{\circ} .57, b=-0^{\circ} .92\right)$ passes twice through the Carina spiral arm (see Fig. 4 of Grabelsky et al. 1988), and we can use information from CO radio emission maps to help place the giant molecular clouds and associated massive star-forming regions along this line of sight. Grabelsky et al. (1988) and Dame et al. (2001) show that there is a large and rare hole in the near side of the Carina spiral arm in this direction that might allow us to see some very distant clusters. This first cut through the arm occurs at a distance of $\approx 2.4 \mathrm{kpc}$, and Shorlin et al. (2004) discuss some of the sparsely populated field stars they find at this distance. The next molecular cloud down the line is found at a distance of $6.8 \mathrm{kpc}$ in the direction $l=290^{\circ} .2, b=-0^{\circ} .2$ (with a radial velocity of $-1 \mathrm{~km}$ $\mathrm{s}^{-1}$ and designated number 14 in Fig. 2 of Grabelsky et al. 1988), which is located about $1^{\circ} .0$ away from the WR 38 cluster. This distance is consistent with the distance error range for the WR 38 cluster and the other nearby WR stars. However, just beyond this, at $7.9 \mathrm{kpc}$, we encounter one of the largest molecular clouds in the Carina spiral arm (with a radial velocity of $+22 \mathrm{~km} \mathrm{~s}^{-1}$ and given as number 13 in Fig. 2 of Grabelsky et al. 1988), where our line of sight crosses the spiral arm a second time. The WR 38 cluster lies only $0^{\circ} .4$ away from the edge of this cloud. There is very little CO emission in the $(l, v)$ diagram corresponding to distances beyond this, so it is doubtful that there are favorable environments for massive star formation beyond a distance of $8 \mathrm{kpc}$ in this direction (see also Fig. 5 in Russeil 2003). However, McClure-Griffiths et al. (2004) present deep radio maps of $21 \mathrm{~cm}$ emission from neutral hydrogen in this region that suggest that some weak emission is present from an extension of the Perseus arm (at a distance of $\approx 15 \mathrm{kpc}$ and a radial velocity of $+50 \mathrm{~km} \mathrm{~s}^{-1}$ ) and a possible outer arm (at a distance of $\approx 21 \mathrm{kpc}$ and a radial velocity of $+120 \mathrm{~km} \mathrm{~s}^{-1}$ ). The outer disks of large spiral galaxies occasionally show clear $\mathrm{H}_{\mathrm{I}}$ spiral arms extending to radii in excess of $30 \mathrm{kpc}$, while the stellar arms stop at radii of about $10 \mathrm{kpc}$. A classic example is M83, in which the $\mathrm{H}$ I spiral arms extend 3 times as far as the stellar arms (Tilanus \& Allen 1993). Note that there are very few WR stars known in the Milky Way beyond the solar circle (see Fig. 6 in van der Hucht 2001), where they would be easy to discover if they existed there (because of generally low reddening). Furthermore, the number ratio of WR to O-type stars decreases dramatically in low-metallicity environments such as the outer Galaxy, so even if there are fair numbers of $O$ stars in the outer Galaxy, there will be relatively fewer WR stars there. 
Georgelin et al. (2000) discuss the stars that are associated with $\mathrm{H}$ II regions in this general direction, and they find that the extinction ranges from $A_{V} \approx 1.8$ at $2.8 \mathrm{kpc}$ to $A_{V} \approx 2.6$ at $4.2 \mathrm{kpc}$, and up to $A_{V} \approx 4$ at $8 \mathrm{kpc}$. Our derived extinction of $A_{V}=5.0 \pm 0.2$ would tend to suggest that the WR 38 cluster also resides at a distance of $\approx 8 \mathrm{kpc}$ or larger. The $8 \mathrm{kpc}$ distance would place the WR 38 cluster approximately midway between molecular cloud number 13 (and the associated H II complex 289.3-0.6; Georgelin et al. 2000) and molecular cloud number 17 ( $\mathrm{H}$ II complex 291.6-0.7), which is associated with the starburst cluster NGC 3603 (Moffat et al. 2002). Note that Sung \& Bessell (2004) find a reddening of $E(B-V)=1.8$ in the outskirts of NGC 3603 that is comparable to that for the WR 38 cluster. Georgelin et al. (2000) identify an $\mathrm{H}$ II region $290.487-0.814$ within the larger 289.3-0.6 complex that has as exciting stars sources 48 and 52 from the list of Wramdemark (1976). The two stars have a comparable extinction $\left(A_{V} \approx 3.7\right)$ and are located only $\approx 0.3$ away from the cluster, so these stars and the $\mathrm{H}$ in region could be related to the WR 38 /WR 38 a cluster.

The solution to the distance problem for the WR 38/WR 38a cluster will come when spectra can be obtained for the cluster stars. Classification dispersion spectra would help establish the temperatures and luminosities of the stars and thus determine accurately the distance modulus of the cluster. Furthermore, radial velocities from spectra would also allow us to distinguish between the differential Galactic rotation expected for the lo- cations along the Carina and Perseus spiral arms. Such observations would clearly help us understand the history of massive star formation in this distant region of the Galaxy.

We are grateful to David Turner for sharing his results in advance of publication and for helpful comments on an earlier version of this work. We also thank Thomas Dame for comments about the molecular emission in this region of the sky. Support for HST proposal numbers GO-6365 and GO-7282 was provided by NASA through grants from the Space Telescope Science Institute, which is operated by the Association of Universities for Research in Astronomy, Inc., under NASA contract NAS5-26555. D. J. W. is also grateful for the support and financial assistance of the Georgia Space Grant Fellowship Program. This research was published while D. J. W. held a National Research Council Associateship Award at the National Aeronautics and Space Administration's Goddard Space Flight Center. A. F. J. M. thanks NSERC (Canada) and FCAR (Quebec) for financial support. This publication makes use of data products from the Two Micron All Sky Survey, which is a joint project of the University of Massachusetts and the Infrared Processing and Analysis Center, California Institute of Technology, funded by the National Aeronautics and Space Administration and the National Science Foundation.
Anderson, J., \& King, I. R. 1999, PASP, 111, 1095

Baggett, S. 2002, HST WFPC2 Data Handbook (4th ed.; Baltimore: STScI)

Bessell, M. S., Castelli, F., \& Plez, B. 1998, A\&A, 333, 231

Bushouse, H., \& Simon, B. 1998, SYNPHOT User's Guide (4th ed.; Baltimore: STScI)

Cohen, M., Wheaton, W. A., \& Megeath, S. T. 2003, AJ, 126, 1090

Cutri, R. M., et al. 2003, The 2MASS All-Sky Catalog of Point Sources (Pasadena: IPAC)

Dame, T. M., Hartmann, D., \& Thaddeus, P. 2001, ApJ, 547, 792

Dolphin, A. E. 2000, PASP, 112, 1397

Ferguson, H. C. 1996, Background Subtraction in WFPC2 Frames, Instrument Science Report 3 (Baltimore: STScI)

Figer, D. F., McLean, I. S., \& Najarro, F. 1997, ApJ, 486, 420

Fitzpatrick, E. L. 1988, ApJ, 335, 703 1999, PASP, 111, 63

Georgelin, Y. M., Russeil, D., Amram, P., Georgelin, Y. P., Marcelin, M., Parker, Q. A., \& Viale, A. 2000, A\&A, 357, 308

Gies, D. R. 1987, ApJS, 64, 545

Gonzaga, S. 2002, WFPC2 Data Analysis: A Tutorial (3rd ed; Baltimore STScI)

Grabelsky, D. A., Cohen, R. S., Bronfman, L., \& Thaddeus, P. 1988, ApJ, 331, 181

Holtzman, J. A., et al. 1995, PASP, 107, 156

Johnson, H. L., Iriarte, B., Mitchell, R. I., \& Wisniewskj, W. Z. 1966, Comm. Lunar Planet. Lab., 4, 99

Johnson, H. L., \& Morgan, W. W. 1953, ApJ, 117, 313

Lanz, T., \& Hubeny, I. 2003, ApJS, 146, 417

Lejeune, T., \& Schaerer, D. 2001, A\&A, 366, 538

MacConnell, D. J., \& Sanduleak, N. 1970, PASP, 82, 80

Maíz-Apellániz, J., Walborn, N. R., Galué, H. Á., \& Wei, L. H. 2004, ApJS, 151,103

Marston, A. P. 1997, ApJ, 475, 188

Mason, B. D., Gies, D. R., Hartkopf, W. I., Bagnuolo, W. G., Jr., ten Brummelaar, T., \& McAlister, H. A. 1998, AJ, 115, 821

Massey, P., DeGioia-Eastwood, K., \& Waterhouse, E. 2001, AJ, 121, 1050

\section{REFERENCES}

McClure-Griffiths, N. M., Dickey, J. M., Gaensler, B. M., \& Green, A. J. 2004 , ApJ, 607, L127

Moffat, A. F. J., et al. 2002, ApJ, 573, 191

Nelan, E., \& Makidon, R. 2002, HST FGS Data Handbook (4th ed.; Baltimore: $\mathrm{STScI})$

Nugis, T., \& Lamers, H. J. G. L. M. 2002, A\&A, 389, 162

Russeil, D. 2003, A\&A, 397, 133

Schönberner, D., \& Harmanec, P. 1995, A\&A, 294, 509

Shara, M. M., Smith, L. F., Potter, M., \& Moffat, A. F. J. 1991, AJ, 102, 716

Shorlin, S. L., Turner, D. G., \& Pedreros, M. H. 2004, PASP, 116, 170

Stetson, P. B. 1987, PASP, 99, 191

Sung, H., \& Bessell, M. S. 2004, AJ, 127, 1014

Tilamus, R. P. J., \& Allen, R. J. 1993, A\&A, 274, 707

Torres, A. V., \& Massey, P. 1987, ApJS, 65, 459

Torres-Dodgen, A. V., \& Massey, P. 1988, AJ, 96, 1076

Turner, D. G. 1976, AJ, 81, 97

Underhill, A. B., Divan, L., Préot-Burnichon, M.-L., \& Doazan, V. 1979, MNRAS, 189, 601

van der Hucht, K. A. 2001, NewA Rev., 45, 135

van der Hucht, K. A., Conti, P. S., Lundstrom, I., \& Stenholm, B. 1981, Space Sci. Rev., 28,227

van Genderen, A. M., Steemers, W. J. G., \& van der Hucht, K. A. 1987, A\&A, 185,131

Wallace, D. J. 2003, Ph.D. thesis, Georgia State Univ., Atlanta

Wallace, D. J., Shara, M. M., Moffat, A. F. J., \& Niemela, V. S. 1999, in IAU Symp. 193, Wolf-Rayet Phenomena in Massive Stars and Starburst Galaxies, ed. K. A. van der Hucht, G. Koenigsberger, \& P. R. J. Eenens (San Francisco: ASP), 112

Wegner, W. 1994, MNRAS, 270, 229

Whitmore, B., Heyer, I., \& Casertano, S. 1999, PASP, 111, 1559

Willis, A. J. 1991, in IAU Symp. 143, Wolf-Rayet Stars and Interrelations with Other Massive Stars in Galaxies, ed. K. A. van der Hucht \& B. Hidayat (Dordrecht: Kluwer), 265

Wramdemark, S. 1976, A\&AS, 23, 231 